\title{
IoT Enabled Smart Waste Bin with Real Time Monitoring for efficient waste management in Metropolitan Cities
}

\author{
Manju Mohan', RM. Kuppan Chetty', Vijayram Sriram², Mohd. Azeem², P. Vishal ${ }^{2}$ \\ and G. Pranav ${ }^{2}$ \\ ${ }^{1}$ Centre for Automation and Robotics (ANRO), School of Mechanical Sciences, Hindustan Institute of \\ Technology and Science, Padur, Chennai - 603103. \\ ${ }^{2}$ B.Tech Mechatronics Engineering, School of Mechanical Sciences, Hindustan Institute of Technology and \\ Science, Padur, Chennai - 603103.
}

\begin{abstract}
Background/Objectives: Waste bins are part of our lives for decades and mostly its condition are overflowing due to improper waste dumping, collection and management, which leads in foul smell and unhygienic condition, thus inherently results in environment pollution. Methods/Statistical analysis: Therefore, in this paper, design of a Waste Bin with real time monitoring is presented and a smart waste management system is proposed using the recent technical advancements of automation and Internet of Things (IoT). Findings: The capacitance sensor in the bin continuously monitors the level of the bin in real time and communicates to the central cloud where the bins are connected. Ultrasonic sensor is used to open and close the lid of the bin whenever the persons are nearby the bin. Such smart bins are connected to the cloud, where the bin status are communicated, recorded and monitored by the local bodies through and android app or a centralized server. Improvements/Applications: Thus the designed smart bin and proposed waste management system have better level of smartness compared to existing ones in metropolitan cities in a centralized manner.
\end{abstract}

\section{Index Terms}

Node Mcu, Internet of Things (IOT), Node Mcu, Smart Waste Bin, Solid waste management, Ultrasonic Sensor

\author{
Corresponding author : RM. KUPPAN CHETTY \\ kuppanc@hindustanuniv.ac.in \\ - Manuscript received July 18, 2019. \\ - Revised August 22, 2019 ; Accepted September 20, 2019. \\ - Date of publication September 30, 2019. \\ (c) The Academic Society of Convergence Science Inc. \\ 2619-8150 @ 2019 IJASC. Personal use is permitted, but republication/redistribution requires IJASC permission.
}




\section{INTRODUCTION}

The smart way of doing things done is the concept behind the smart city's development with less man power and maximum utilization of the technology in the day today life. The use of technologies is very significant towards the development of smart cities. Automation is the key part in the smart cities irrespective of various needs and applications [1]. At places, whether it is a rural or urban area or apartment or multi storey buildings, there occurs the accumulation of wastes. The applicability of a proper waste management system is significant at this point. Rise in urbanization and increase in population matches up to the quantity of solid waste in today's life [2, 3]. Therefore, solid waste management and managing the condition of the waste bins possess a major challenge in metropolitan cities like Chennai, India. Waste bins are part of our lives for decades and mostly the condition of the waste bins is overflowing due to improper waste dumping, collection and management leads in foul smell and unhygienic condition which inherently results in environment pollution.

The old-fashioned method of management of solid waste is an unwieldy and intricate process, which utilizes enormous human effort, valuable time and hefty cost and is not well matched with advancement in technologies. One of the smart ways of monitoring the things is the use of "Internet of Things" (IoT). Through IoT, devices with internet connectivity can be monitored and controlled remotely. We have proposed a waste management system by utilizing the concept of IoT to connect the different dust bins at different locations and also to identify the level of waste in a dust bin through a centralized system. Hence, the concerned persons will be notified about each bin status and there by achieving the removal of wastes in a proper way.

A smart bin for waste management is designed using an Arduino microcontroller, Wi-fi module and sensors to indicate the level of waste, to detect harmful gas and closing of lid thereby giving a solution to collect the waste in proper way [4]. Real time monitoring and collection of solid waste for smart city services is addressed in [5], where each bin is installed with Arduino microcontroller, ultrasonic sensor and Radio Frequency (RF) transmitter on the top of the container which sends signal to the control center through GSM/GPRS when the bin is filled [5].

Smart Garbage Management System is developed and addressed in [6] where, IR sensors embedded on dustbins for waste level detection, GSM 900 modem is used to send waste level data collected by microcontroller, with an additional graphical user interface using MATLAB software. A smart waste management system is proposed by an on-site and real time monitoring of waste as well as a data elaboration through decisional algorithms [7]. An automatic smart waste management system is presented in [8], where smart vehicle system with a local base station and a trash system with smart monitoring \& controlling hut having two load sensor and IR proximity sensor were used and addressed.

In [9] a step had been taken to connect the various sensor or actuators in a network through an Access Point (AP) to the cloud and investigated on three different sensor applications. Power consumption, Interference impact and range performance analysis are also evaluated for each application and discussed. Smart community is an emerging application of technological advancement of IoT. Smart community architecture is defined and realized by connecting individual homes through IoT. A similar application of the smart community in Neighborhood Watch and Pervasive Healthcare is presented and challenges involved are also discussed in [10]. A possibility of using Mobile-2-Mobile (M2M) solutions for management of road traffic linking IoT is investigated in [11]. The use of Industrial Wireless Sensor Networks (WSN) in IoT environment is also proposed in [12]. In this work, the design of a smart sensor interface by connecting the sensors in WSN allowing the reconfigurability by reading the data in parallel as well as in real time using ARM Controller is also discussed in detail. Similarly, a smart home control network is developed and evaluated for the smart control of lighting systems in smart homes by using a scalable architecture combining WSN and Power line communications (PLC) technologies. This also results in less radio interference and allows an easy replacement of nodes in a WSN [13].

An IoT-based Smart Garbage system (SGS) is reported in [14] which are operated in Gangnam district, Seoul for a period of one year resulting in the reduction of food waste by $33 \%$. Battery based smart dust bins are connected in wireless mesh network and a router and server is used to transfer the information collected in this work. IoT architecture for optimized waste management in smart cities is also realized in [15], where LoRa LPWAN (Low Power Wide Area Network) technology is used for the transmission of data collected from the microcontroller connected ultrasound sensor nodes.

A Spatial Smart Waste Management System (SWMS) is implemented in Malaysia in order to manage the wastes by giving alerts about the waste level in a bin to the contractor for optimizing the collection routes and penalizes if not collected the garbage on time [16]. A smart recycle bin based on 
IoT and $\mathrm{Wi}-\mathrm{Fi}$ is also introduced based on reduce, reuse and recycle concept. It is like an enforcement system that makes the people to classify the waste for recycling and also used DeviceBit and Blynk applications for real time monitoring [17]. A step is taken towards the detailed analysis of various waste management models and an IoT based reference model is implemented and compared with the existing models to identify the best choice and research challenges [18].

According to the literature found and discussed numerous efforts were made in solid waste management and in IoT. However, the major challenge is to bring together the best method of Solid waste management and the technical advancements of IoT. Therefore, In general, a smart waste monitoring system consists of sensors, transmission medium, waste level data acquisition and collection system and connectivity to the cloud has to be found inside a waste bin for having smartness in the system. Inspired from the literature and the Swachh Bharat initiative of the Government of India, the main objective of this paper is to design and develop an IOT Enabled Smart Waste Bin with Real Time Monitoring for efficient waste management system. The developed system intend to reduce valued human resources effort, time and cost as well as to protect the environment and healthy living of the people with the help of Modern technologies such as cloud system, Wi-Fi, ultrasonic ranger sensor, capacitive action.

\section{Methodology}

Inspired from the literature and as a preliminary study, design of a smart waste bin is presented in this section. This section also describes the use of different sensors, actuators and controllers associated with the design of the smart bin. The waste level detection using the capacitance method is also presented. Further, a smart waste management system utilizing the designed smart bins is also addressed as a proposed system using the recent technical advancements of automation and the Internet of Things (IoT).

\section{A. Design of Smart Dust Bin}

Figure 1 shows the block diagram of a smart waste bin, where the smartness is achieved by having ultrasonic sensors, capacitive sensors, microcontrollers, servo motors, NodeMcU and integrating to form a complete autonomous system. The waste level inside the bin is determined by estimating the distance between the bottom of the bin and the lid using ultrasonic sensor. In addition to this, the level of waste is also estimated by using

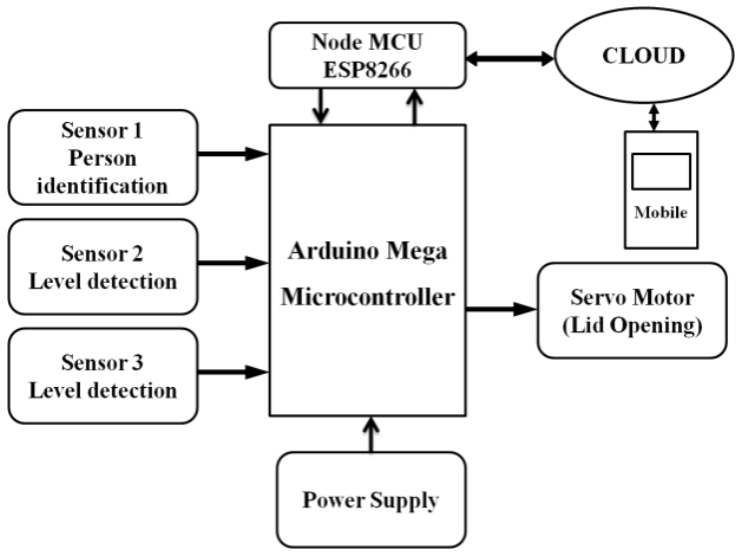

Fig. 1. Block diagram of Smart Dust Bin.

Change in capacitance principle by having parallel plates inside the bin. The sensors are connected with the controller and the levels are continuously recorded in the cloud. When the waste level exceeds the threshold value, which is set according to the dimension of the bin, the controller alerts the responsible municipal persons or the sanitary inspector with the bins ID, bin location and the bin level. An automated opening and closing of the bin lid is also incorporated by having another ultrasonic sensor, which opens the lid of the bin whenever it perceives a person nearby to the bin. A power supply unit provides a necessary power to the operation of the bin. Once the bin is emptied and serviced, it returns to the default operation.

A commonly used cylindrical plastic trash bin with the dimension of $30 \times 20 \times 60 \mathrm{~cm}$ with lid is used to develop the smart bin. As shown in figure 1, the smart bin uses two SRF 04 ultrasonic transceivers for the detection of waste level and to perceive the motion of a person nearby bin respectively. The ultrasonic sensors are operated with the frequency of $40 \mathrm{KHz}$ and the time of flight method is utilized to estimate the level of waste inside the bin and also to estimate the motion of the person when he falls inside the threshold value of $30 \mathrm{~cm}$. Ultrasonic sensor 1 is mounted facing the bottom of the bin, which measures the level of waste inside the bin with the threshold of $25 \%, 50 \%, 75 \%$ and $90 \%$ of the bin depth of $60 \mathrm{~cm}$. Similarly, ultrasonic sensor 2 used to detect the motion of the person is mounted at the top of the bin facing outside. TowerPro MG995 Metal Gear Servo Motor with $180^{\circ}$ operation is used as the actuator for opening and closing the lid of the bin whenever the sensor perceives the nearby motion of the person. The lid closes automatically when the person moves away from the threshold distance. A rechargeable Lithium Polymer (LiPo) battery of $2200 \mathrm{mAh}, 11.1 \mathrm{~V}$ is used as a power supply to the entire system. Entire electronic system is mounted within a weather proof casing and fixed in the trash bin. 
An Atmega2560+NodeMCU ESP8266 microcontroller with $32 \mathrm{Mb}$ (megabits) of flash memory, working on arduino development environment is used as a controller to integrate the sensors, actuators and communication devices to provide the necessary actions required by the smart bin. The advantage of using this controller is that the all of the modules can work together or each separately, eliminating the interface issues, when compared to the other arduino controller used in the literatures. NodeMcU is used as the communication device which connects the system with the IoT cloud by broadcasting the level of waste in bin, status of the bin along with the bin ID and location. Further, it also broadcasts the alert signal to the sanitation inspector or central municipal server whenever the bin exceeds the $90 \%$ threshold level limits. Universal Datagram Protocol (UDP) is used as the communication standard by the system, with the maximum packet size of 255 bytes. However, only 40 bytes are used as a packet size out of which 8 bytes are used as UDP header and remaining 32 bytes for data to effectively communicate the status information to the cloud as shown in Figure 2. The data byte consists of Bin ID, Location with Latitude and Longitude information, Bin Level, Status and Power level.

\begin{tabular}{|c|c|c|c|c|c|c|c|c|c|c|c|c|}
\hline 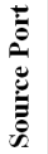 & 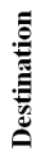 & 䓌 & 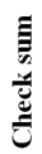 & 音 & 苞 & 串 & 实 & 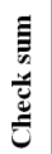 & 总 & 寞 & 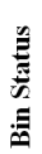 & 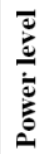 \\
\hline 2 & 2 & 2 & 2 & 2 & 4 & 8 & 4 & 2 & 4 & 4 & 2 & 2 \\
\hline \multicolumn{4}{|c|}{$\begin{array}{c}\text { UDP Header } \\
\text { (8 bytes) }\end{array}$} & \multicolumn{9}{|c|}{$\begin{array}{l}\text { UDP DATA } \\
\text { (32 bytes) }\end{array}$} \\
\hline
\end{tabular}

Fig. 2. Universal Datagram Protocol (UDP) packet.

As mentioned in the previous section, the entire process is developed in Arduino IDE. Figure 3 shows the flowchart of the arduino program where the motion detection and level measurement is executed in parallel.

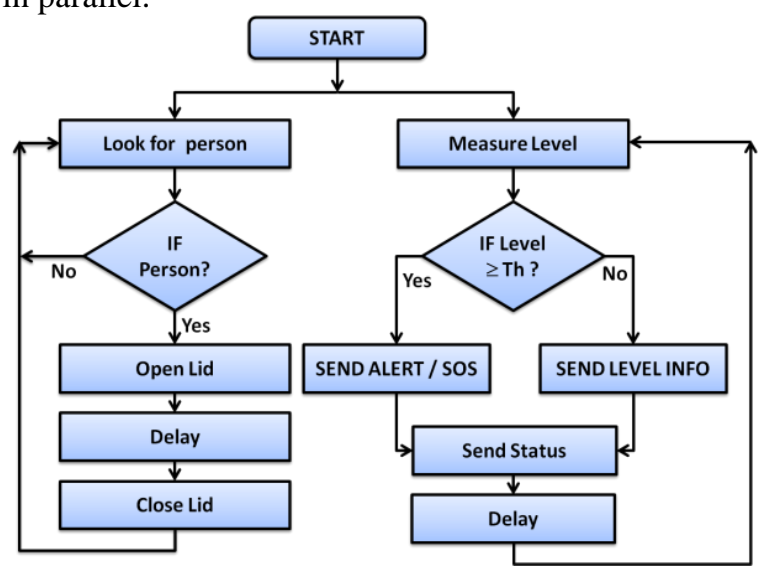

Fig. 3. Software flowchart of Smart Waste Bin
In order to have efficient communication method as per UDP protocol each bin is identified by dedicated IP address configured in the NodeMcU and updates the information of the bin to the cloud for predetermined minutes establishing the connection with the cloud using polling strategy. Polling strategy is utilized to route the data efficiently to the cloud, since the NodeMcU has limited memory and energy constrained, thereby reducing communication overload. As the data are logged into the IoT cloud, the level and status of the bins along with the alerts are monitored using the blynk mobile app installed in the sanitation inspector and the centralized municipal server. Once the bins are serviced and the wastes are removed, the system returns back to the original state.

\section{B. Parallel Plate Capacitor based detection of waste level.}

Most of the design for smart waste bin found in the literature uses ultrasonic sensors to detect the level of waste in the bin which were very similar to the technique discussed in the previous sections and as mentioned in figure 4 (a). However, this level detection is prone to errors while opening the lid of the waste bin due to the motion of the person near the bin to dump the trash. The lid opening causes the sensor to move away from the sensing field of view as depicted in figure 4 (b), and provides faulty data to the cloud and a municipal sanitary inspector. In order to avoid such errors, the level of waste detection is achieved using a simple parallel plate capacitance principle as given in figure 4. Two copper plates of dimension $50 \mathrm{~cm} \times 5 \mathrm{~cm} \times 0.3 \mathrm{~cm}$ is used as the electrodes and mounted opposite to each other, in the wall of the bin as shown in figure 4 . These plates are with the cross sectional area of $250 \mathrm{~cm}^{2}$ and separated with the distance of $18 \mathrm{~cm}$, with the initial capacitance value of $1.22 \mathrm{pF}$ by general capacitance equation given as equation 1, considering the permittivity of free space and air as the dielectric medium between the plates assuming there are no wastes in the bin.

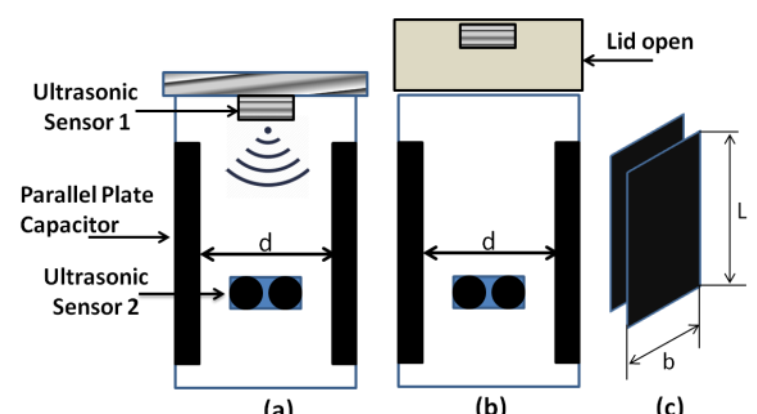

Fig. 4. (a) Smart bin with lid closed and parallel plates (b) Bin with lid open and position of sensor (c) Parallel plates as capacitors 


$$
C=\varepsilon_{y} \varepsilon_{0} \frac{A}{d}
$$

Where,

' $C$ ' is the capacitance between the parallel plates, ' $\varepsilon_{0}$ ' is the permittivity of the free space which is $8.854 \times 10^{-12}$

' $\varepsilon_{\mathrm{r}}$ ' is the relative permittivity or dielectric constant of the material between the plates,

' $\mathrm{A}$ ' is the cross sectional area of the plates

' $d$ ' is the separation distance between the plates.

As the wastes are dumped in the bin the, the waste / trash acts as a dielectric medium between the plates and the capacitance between the plates changes due to the changes in the relative permittivity $\left(\varepsilon_{\mathrm{r}}\right)$ of the material. As a result, this change in capacitance value is correlated to the level of waste inside the bin.

Figure 5 shows a signal conditioning circuit developed to convert the change in capacitance value to electric signal which could be read by the microcontroller for further processing. This circuit consists of two stages where the first stage is the sensing and transduction stage in which the capacitance is converted into the electrical signal by measuring the voltage drop across the capacitor which is given by equation 2 .

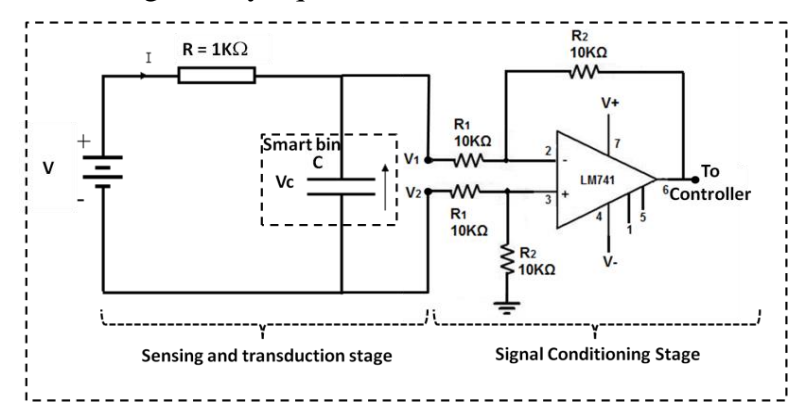

Fig. 5. Sensing, transduction and signal conditioning circuit used to convert capacitance into electrical signal

$$
V_{C}=V\left(1-e^{-t_{R C}}\right)
$$

Where,

' $\mathrm{V}$ ' is the applied voltage

' $\mathrm{Vc}$ ' is the voltage drop across the capacitor

' $R$ ' is the resistance value

' $\mathrm{C}$ ' is the capacitance of the parallel plates,

' $t$ ' is the elapsed time.

As the change in capacitance due to change in the level of dielectric constant, is very small, the voltage drop across the capacitance is also very small, which has to be amplified so that the microcontroller can read the electrical values. Therefore, the second stage of the signal conditioning circuit has a differential amplifier designed using op-Amp LM741/OP07, with appropriate gain values. Hence, the voltage across the capacitor is amplified and fed to the micro controller for the detection of trash level in the bin. This method of detection of waste level is simple and cost effective when compare to the use of ultrasonic sensors

\section{EXPERIMENTS AND RESULTS}

Design and development of an IoT enabled smart waste bin using parallel plate capacitance and ultrasonic sensor based level detection is presented and discussed in the previous section. Series of experiments were carried out to measure the performance of the waste bin. Experiments were conducted by establishing a cloud linking the waste bins with the local area network in our centre at Hindustan Institute of Technology and Science. Experiments are conducted using plastic bottles and paper as trash. The change in capacitance is estimated to be $4.6 \mathrm{pF}$, when the bin is full of plastic bottles as trash and $2.2 \mathrm{pF}$ for the full bin with paper as trash. Figure 6 shows the value of capacitance obtained theoretically as well as experimentally for different levels of the bin. The statuses of the bins are monitored using the blynk app, running in the mobile phone.

Figure 7, depicts the qualitative results of the statuses of the bin monitored using blynk app. The level of waste in the bin is represented by traffic lights in the app where red indicates the fullness of the bin, green indicates the $25 \%$ level and yellow indicates the $50 \%$ level. It could be observed from figure 5 , that whenever a motion is detected near to the bin, the bin lid opens automatically and the level of the bin is detected, communicated to the cloud and displayed in the app effectively.

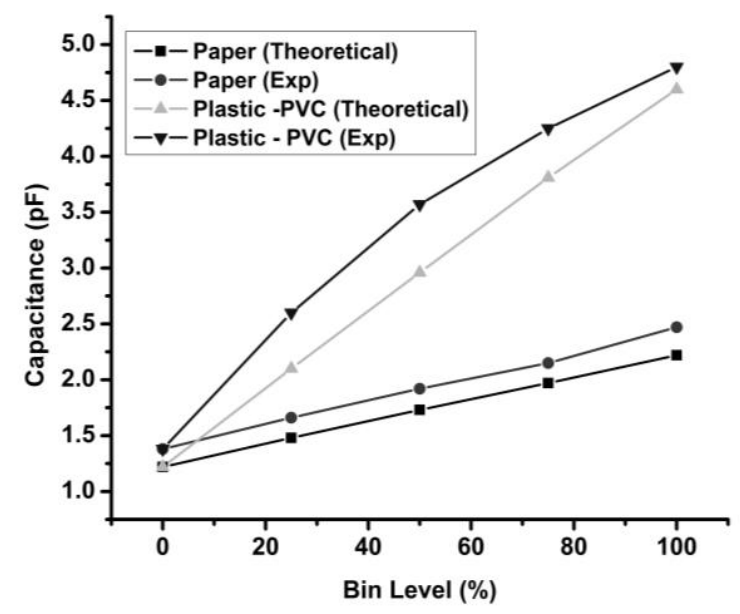

Fig. 6. Capacitance values for different bin levels for paper and plastic-PVC as trash.

\section{Proposed system}

Figure 8 shows the general architecture which uses the cluster of smart waste bins connected through IoT in the outdoor environment as mentioned in the previous sections. It uses a GPS, GSM/GPRS modules instead of the NodeMcu's to 
communicate the status of the smart waste bins enabling the effective waste management system,

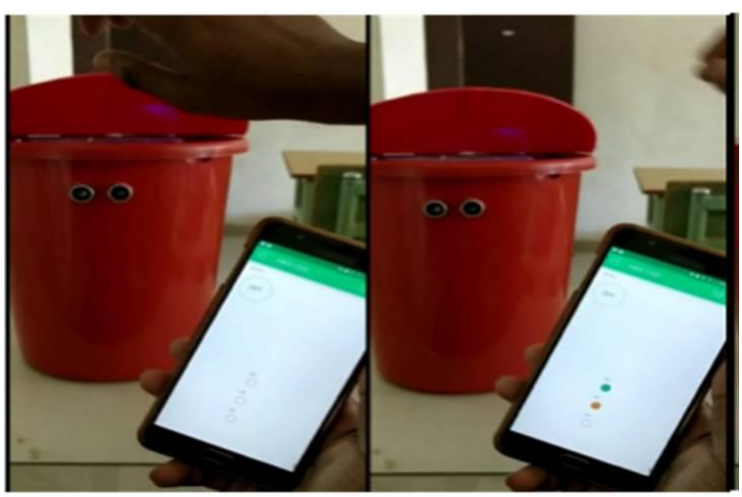

(a)

(b)

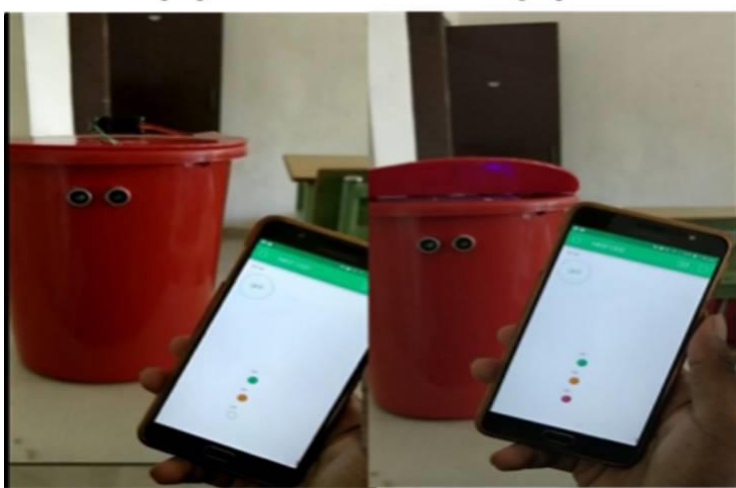

(c)

(d)

Fig. 7. (a) Detection of motion and opening of lid with bin empty, (b) bin is $50 \%$ full and lid open, (c) bin is $50 \%$ full and lid is in closed condition when motion is not detected (d) Alert due to the bin is with full of waste.

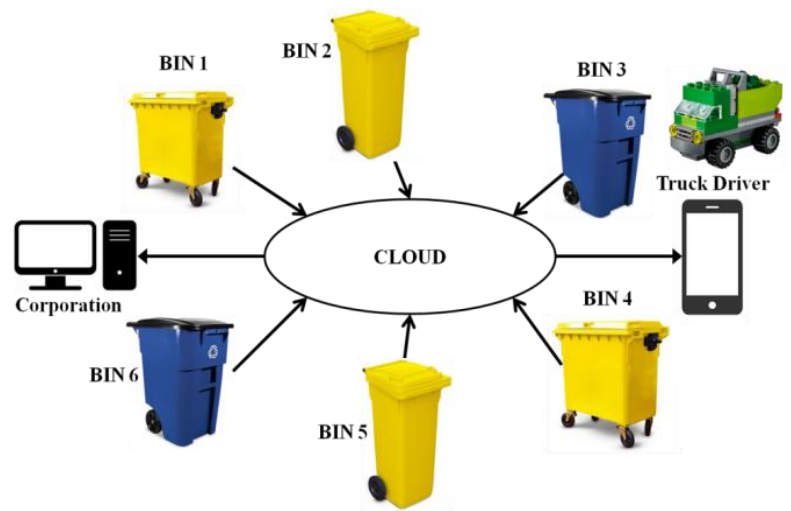

Fig. 8. Architecture of the waste management system using smart waste bins and IoT.

Which is planned to be implemented in our Hindustan Institute of Technology and Science campus. Figure 9 depicts our proposed architecture similar to the one illustrated in figure 6 . The major difference is that the smartness of the waste bin is improved by adding the mobility for the bins to move from its location through following guide lines to the common waste management dump yard rather than indicating to the sanitary worker to service it.

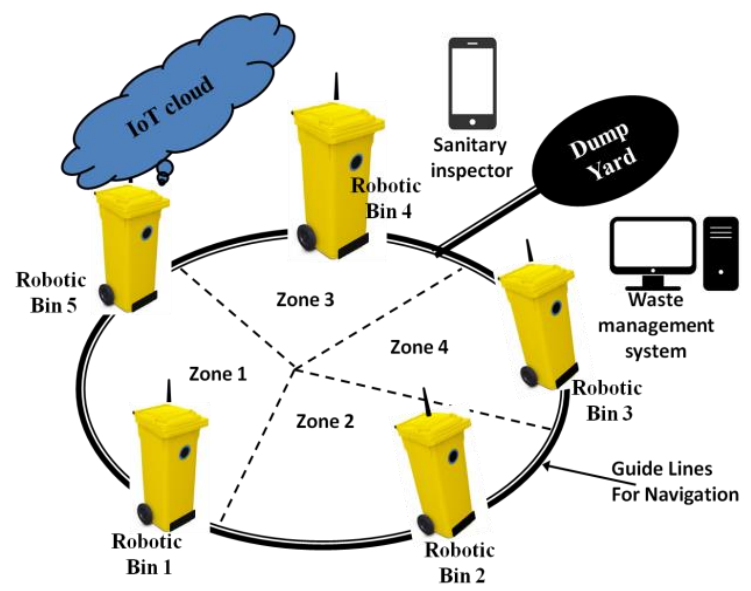

Fig. 9. Architecture of the indoor waste management system using robotic smart waste bins and IoT.

Therefore, the proposed system could be considered as a robotic smart waste bin, where the bins could mobilize, localize its location; communicate its status to the cloud. During the bins are serviced at the dump yard, spare bins could automatically replace the serviced bins enhancing the effectiveness of the system. This concept is proposed due to the evolution of the smart buildings and smart homes in recent times and it could be a potential technical advancement in the area of waste management systems and IoT.

\section{Conclusion}

In most of the metro cities globally poses a challenge on effective waste solid waste management and maintenance of the waste bins. In this work an IOT enabled Smart Waste Bin with real time monitoring is designed and presented. In addition to the waste level measurement by using ultrasonic sensors, a sensing mechanism based on simple parallel plate capacitance is also developed and presented. Experimental investigations are carried out where the waste level of the smart bins is measured using the parallel plate capacitance and ultrasonic sensors and the statuses of the bins are communicated to the cloud effectively. The results prove the efficiency of the designed smart bins qualitatively. A smart waste management system incorporating robotic smart bins, where the smart bin has the mobility to move to the waste dockyard by localizing itself in the environment, is also proposed in this work. This system could find an application in smart buildings where the waste management could be practiced autonomously in a smarter way. Our future work is to investigate the performance of the proposed traditional and robotic waste management system in outdoor and indoor environment respectively in our Institutional campus. 


\section{ACKNOWLEDGMENT}

Authors wish to thank Centre for Automation and Robotics, School of Mechanical Sciences, for the support and lab space provided to carry out this work under the Research Incubation Program, of Hindustan Institute of Technology and Science, Padur, Chennai - 603103.

\section{REFERENCES}

[1] Hitesh Poddar, Rituraj Paul, Sourangsu Mukherjee \& Budhaditya Bhattacharyya. (2017). Design of smart bin for smarter cities. In. IEEE Proceedings of Innovations in Power and Advanced Computing Technologies (i-PACT), Vellore.1-6.

[2] Rajkumar Joshi \& Sirajuddin Ahmed. (2016). Status and challenges of municipal solid waste management in India: A review. Cogent Environmental Science, 2: 1139434, 1-18.

[3] Eunice Likotiko, Dmitry Petrov, Joseph Mwangoka \& Ulrich Hilleringmann. (2018). Real time solid waste monitoring using cloud and sensors technologies. The Online Journal of Science and Technology, 8(1), 106-116.

[4] Sreejith S, Ramya R, Roja R. \& Sanjay Kumar A. (2019). Smart Bin for Waste Management System. In. Poceedings of the IEEE $5^{\text {th }}$ International Conference on Advanced Computing \& Communication Systems, Coimbatore, India, 1079-1082.

[5] Hassan, S. A., Jameel, N.G.M. \& Boran. S. (2016). Smart Solid Waste Monitoring and Collection System. International Journal of Advanced Research in Smart Solid Waste Monitoring and Collection System, 6(10), $7-12$.

[6] Bhor, V., Morajkar, P. \& Amol Deshpande. (2015). Smart Garbage Management System. International Journal of Engineering Research \& Technology (IJERT), 4(3), 1117-1120.

[7] Catania, V. \& Ventura, D. (2014). An approach for monitoring and smart planning of urban solid waste management using smart-M3 platform. In Proceedings of the IEEE 15th Conference of Open Innovations Association FRUCT, Saint Petersburg, Russia, 24-31.

[8] Bashir, A., Banday, S. A., Ab. Rouf Khan \& Mohammad Shafi. (2013). Concept, Design and Implementation of Automatic Waste Management System. International Journal on Recent and Innovation Trends in Computing and Communication, 1(7), 604-609.

[9] Serbulent Tozlu; Murat Senel; Wei Mao. \& Abtin Keshavarzian. (2012). Wi-Fi enabled sensors for internet of things: A practical approach. IEEE Communications Magazine, 50(6), 134-143.

[10] Xu Li, Rongxing Lu, Xiaohui Liang, and Xuemin (Sherman) Shen, Jiming Chen \& Zhejiang Xiaodong Lin. (2011). Smart Community: An Internet of Things Application. IEEE Communications Magazine, 49(11), 68-75.

[11] Luca Foschini, Tarik Taleb \&Antonio Corradi. (2011). M2M-based metropolitan platform for IMS-enabled road traffic management in IoT," in IEEE Communications Magazine, 49(11), 50-57.
[12] R. Prakash More \& Anil. S. Hiwale. (2016). A Reconfigurable Smart Sensor Interface for Industrial WSN in IoT Environment. International Journal of Innovative Research in Science, Engineering and Technology, 5, 8986-8994.

[13] M. Li \& H. Lin. (2015). Design and Implementation of Smart Home Control Systems Based on Wireless Sensor Networks and Power Line Communications. In. IEEE Transactions on Industrial Electronics, 62(7), 4430-4442.

[14] I. S. Hong, S. H. Park, B. S. Lee, J. K. Lee, D. B Jeong \& S. H. Park. (2014). IoT-Based Smart Garbage System for Efficient Food Waste Management. The Scientific World Journal, 2014, 646953, 1-13.

[15] Cerchecci M, Luti F, Mecocci A, Parrino S, Peruzzi G \& Pozzebon A. (2018). A Low Power IoT Sensor Node Architecture for Waste Management within Smart Cities Context. Sensors (Basel), 18(4), 1282, 123.

[16] M. F., Omar, A.A. A., Termizi, D., Zainal, N. A., Wahap, N. M., Ismail \& N., Ahmad (2016). Implementation of spatial smart waste management system in Malaysia. In IOP Conf. Series: Earth and Environmental Science, 37, 012059, Malaysia.

[17] Noor Salah \& Rabee M.Hagem. (2018). Smart Recycle Bin System based on Wi-Fi and IoT. International Journal of Computer Applications, 181(4), 34-37.

[18] Kellow Pardini, Joel J. P. C. Rodrigues, Sergei A. Kozlov, Neeraj Kumar \& Vasco Furtado. (2019). IoTBased Solid Waste Management Solutions: A Survey. Journal of Sensor and Actuator Networks, 8(1), 5. 